\title{
Ability of a high-total antioxidant capacity diet to increase stool weight and bowel antioxidant status in human subjects
}

\author{
Marta A. Bianchi ${ }^{1}$, Francesca Scazzina ${ }^{1}$, Daniele Del Rio ${ }^{1}$, Silvia Valtueña ${ }^{2}$, Nicoletta Pellegrini ${ }^{1}$, \\ Laura Franzini $^{2}$, Maria Luisa Callegari ${ }^{3}$, Claudia Pellacani ${ }^{4}$, Annamaria Buschini ${ }^{4}$, Ivana Zavaroni ${ }^{2}$ \\ and Furio Brighenti ${ }^{1 *}$ \\ ${ }^{1}$ Department of Public Health, University of Parma, Via Volturno 39, 43100 Parma, Italy \\ ${ }^{2}$ Department of Internal Medicine and Biomedical Sciences, University of Parma, Parma, Italy \\ ${ }^{3}$ Centro Ricerche Biotecnologiche (CRB), Cremona, Italy \\ ${ }^{4}$ Department of Genetics, Biology of Microorganisms, Anthropology, Evolution, University of Parma, Parma, Italy \\ (Received 28 January 2010 - Revised 17 May 2010 - Accepted 20 May 2010 - First published online 28 June 2010)
}

\begin{abstract}
There is limited knowledge about the possible effect of unabsorbed dietary antioxidants that reach the large intestine on bowel habits. The aim of the present study was to investigate whether a dietary recommendation directed to increase diet total antioxidant capacity (TAC) is able to affect gut function in human subjects. In this cross-over intervention, nineteen subjects followed a high-TAC (HT) and a low-TAC (LT) diet for 2 weeks, which were comparable for energy, macronutrient, total dietary fibre and alcohol contents. At the end of each intervention period, the $48 \mathrm{~h}$ stool output was recorded. In the faecal samples obtained from a subset of nine subjects, moisture, $\mathrm{pH}$, ammonia content, Lactobacillus and Bifidobacterium counts, faecal water antioxidants and genotoxicity were measured. A $3 \mathrm{~d}$ weighed food record was used to assess the diet composition during HT and LT diet intake. Significant increases in the intake of TAC, vitamins E and C and phenolic compounds were observed during the HT diet intake. The higher intake of antioxidants led to increased $48 \mathrm{~h}$ stool output (324 (SD 38) g in HT v. 218 (SD 22) g in LT), and to higher TAC and total phenolic concentrations in faecal water. No significant variation in the other measured parameters was observed between the diets. In conclusion, a diet selected to raise the intake of dietary antioxidants is able to increase stool bulk and antioxidant content of faeces.
\end{abstract}

Total antioxidant capacity: Stool weight: Bowel function: Antioxidant status: Dietary antioxidants

Human diets greatly differ in their effects on bowel function and, possibly, health. Plant foods, especially fruit, vegetables, whole-grain cereals and pulses, are the main dietary sources of functional compounds such as vitamin and non-vitamin antioxidants and indigestible carbohydrates, and represent the main pillars of dietary strategies aiming to reduce the risk of diet-related chronic diseases, namely type 2 diabetes, CVD and cancer ${ }^{(1-3)}$, including colon cancer. Since the introduction of the dietary fibre hypothesis in the early 1970s, indigestible bulk carbohydrates such as cellulose and hemicelluloses, and more recently prebiotic oligosaccharides, have been considered the components responsible for the beneficial effects of plant foods on bowel function. Bowel habit, a marker of bowel function, is usually defined in terms of frequency of defaecation, stool consistency and form, and stool weight. In particular, dietary fibre is able to increase stool weight, whereas low stool weight has been associated with increased risk of colon cancer, diverticular disease, appendicitis and various rectal conditions ${ }^{(4,5)}$.

Little is known about the effects of non-carbohydrate plant components with antioxidant activity on bowel habits.
Dietary antioxidant compounds are generally poorly digested and absorbed in the small intestine, and therefore enter the colon. On one hand, stool weight has been associated to the consumption of coffee and polyphenol-rich fruits and vegetables in observational studies ${ }^{(6)}$, and to the consumption of tea in human interventions ${ }^{(7)}$. On the other hand, interventions with foods rich in antioxidant vitamins and carotenoids, such as carrot or tomato juice, do not appear to influence the faecal mass ${ }^{(8)}$, possibly due to the higher bioavailability of these antioxidant compounds in the upper intestine ${ }^{(9)}$. In human subjects, it is estimated that appoximately $400-570 \mathrm{mg} / \mathrm{d}$ of phenolic acids, polyphenols and tannins (such as aglycones) enter the colon ${ }^{(10)}$, and significant concentrations have been quantified in human faecal water ${ }^{(11)}$. In rats, poorly absorbed polyphenols, such as highly bound or condensed tannins, can increase water, fat and protein excretion, leading to increased faecal weight ${ }^{(12,13)}$.

The total antioxidant capacity (TAC) is a parameter that is able to describe the concentration and synergy of antioxidants both in foods and in biological samples ${ }^{(14,15)}$, and it is especially related to their content in phenolic compounds ${ }^{(16)}$.

Abbreviations: HT, high-total antioxidant capacity; LT, low-total antioxidant capacity; RT-PCR, real-time PCR; TAC, total antioxidant capacity; WO, wash-out. * Corresponding author: F. Brighenti, fax +390521 903832, email furio.brighenti@unipr.it 
To our knowledge, and despite animal experiments and observational human studies indicating that supplementation with antioxidants can increase the antioxidant content in faeces ${ }^{(6,17)}$, no data exist from intervention studies conducted in human subjects linking dietary TAC and markers of bowel function. Therefore, the present trial was conducted with the primary objective of investigating whether the choice of foods with high TAC (HT) modifies markers of bowel function such as stool output, evacuation frequency and stool consistency, while controlling for the total number of plant food servings and amount of total dietary fibre. Lactobacillus and Bifidobacterium counts, antioxidant concentration and genotoxicity of faecal waters were also assessed to evaluate the effects of dietary TAC on these diet-modifiable biomarkers previously reported as potentially related to gut health ${ }^{(18-20)}$.

\section{Experimental methods}

\section{Subjects}

Thirty-four adults from a cohort of apparently healthy workers and ex-workers of a local food company who were enrolled in a follow-up survey on risk factors for diabetes and CVD volunteered to participate in an intervention study on the effects of HT diets on inflammation and liver function ${ }^{(21)}$. Exclusion criteria were diabetes mellitus, cardiovascular events, evidence of hepatitis $B$ virus or hepatitis $C$ virus infection, chronic liver diseases or nephropathies, cancer, organ failure, smoking, last menses within the past 12 months, use of cholesterol-lowering or anti-inflammatory medications, and hormone replacement therapy for the past 12 months.

Among these, twenty subjects accepted to participate in the present study by undergoing, in addition to the original protocol, further investigations regarding bowel function and stool composition. Additional exclusion criteria for this subgroup were history of gastrointestinal diseases, family history of colorectal cancer (first-/second-degree relatives), gluten or lactose intolerance, antibiotic therapy within the last 3 months, use of laxatives or drugs affecting the gastrointestinal tract motility. Nine complied completely with the protocol and provided faecal samples for the analysis, whereas eleven provided dietary records and diaries on bowel function only.

The present study was conducted according to the guidelines laid down in the Declaration of Helsinki, and all procedures involving human subjects were approved by the Ethics Committee for Human Research of the University of Parma. Written informed consent was obtained from all the subjects.

\section{Study design, diet and dietary records}

The study design has been described in detail elsewhere ${ }^{(21)}$. In short, subjects participated in a randomised cross-over study in which they followed a HT and a low-TAC (LT) diet for 2 weeks each, with a $14 \mathrm{~d}$ wash-out (WO) period in between. The HT and LT diets were designed to substantially differ in the amount of TAC while providing similar energy, macronutrients, total dietary fibre and alcohol. Dietary instructions focused on the alternative choice of HT or LT food items (lists of allowed HT or LT fruits, vegetables, beverages, sweets, condiments and alcoholic beverages were made available to the participants, as described in Valtueña et al. ${ }^{(21)}$ ), with the specific request of consuming five medium-sized portions of fruits and vegetables daily.

To control for dietary sources of TAC and to enhance compliance, a wide choice of food items permitted during each dietary period was delivered biweekly to the subjects at home free of charge and in sufficient amounts to cover the intended consumption of each subject and his or her household. Subjects were also instructed to follow suggestions regarding the consumption of first courses, with particular attention being paid to seasoning (i.e. use of tomato sauce, olive oil, vinegar and spices). Finally, the subjects were asked to consume their usual diet during the WO period, and to maintain their usual dietary habits relative to the consumption of meat, fish, milk and dairy products, eggs, cereal products, sweets, cakes and alcohol throughout the whole 6-week study period.

During the second week of each dietary period, subjects recorded their food consumption in a $3 \mathrm{~d}$ weighed food record. Nutrient and TAC intake data were decoded by referring to the European Institute of Oncology food composition database ${ }^{(22)}$ integrated with the TAC values of more than 150 raw foods ${ }^{(14,15)}$ measured by the ferric reducing antioxidant power assay ${ }^{(23)}$. Polyphenol intake was assessed using the polyphenol food content of the United States Department of Agriculture database (www.ars.usda.gov/Services/ docs.htm?docid $=6231$, accessed June 2008).

Subjects underwent a medical examination at baseline and at weeks 2, 4 and 6, and were asked about diseases that had eventually occurred and medication that were taken in the last 2 weeks. Subjects were also requested not to change their habitual level of physical activity and not to consume food supplements throughout the duration of the study.

\section{Intestinal habits and faecal collection}

Intestinal habits were recorded throughout the 6 weeks of the study by means of an ad hoc diary designed to retrieve data on stool frequency (number of evacuations/d) and consistency, abdominal pain, intestinal bloating and flatulence.

Total faecal output was recorded as total weight of faeces excreted over $48 \mathrm{~h}$ on the first and last days of each dietary period. Subjects were instructed to weigh the samples with the accuracy of the first decimal on electronic scales provided by the staff. Subjects were also asked to collect the faecal samples and deliver them refrigerated within $2 \mathrm{~h}$ of evacuation to the laboratory. Only nine subjects were able to comply with this part of the protocol. Faecal samples were stored at $-20^{\circ} \mathrm{C}$ until further processing.

\section{Faecal sample processing}

Samples were defrosted at room temperature, and $48 \mathrm{~h}$ samples obtained from each subject were pooled together, diluted 1:1 with PBS (Dulbecco's 1X; Applichem GmbH, Darmstadt, Hesse, Germany) and homogenised $(2 \times 2 \mathrm{~min})$ in a Stomacher (Seward Stomacher 200; Seward Limited, Littlehampton, West Sussex, UK). The $\mathrm{pH}$ of the homogenates was measured, and $1 \mathrm{ml}$ aliquots were stored at $-80^{\circ} \mathrm{C}$ until further analysis. Simultaneously, an aliquot $(30 \mathrm{ml})$ of the homogenates was transferred into polypropylene tubes and 
centrifuged $\left(50000 \mathrm{~g}, 2 \mathrm{~h}, 4^{\circ} \mathrm{C}\right)$ using a high-speed refrigerated centrifuge (3K-30; Sigma Laboratory Centrifuge, Osterode, Saxony, Germany). One millilitre of the supernatant, representing the faecal water fraction, was stored at $-80^{\circ} \mathrm{C}$ until further analysis.

\section{Chemical analyses}

Ceramic crucibles were used to assess faecal moisture by oven-drying $0.5 \mathrm{~g}$ aliquots of thawed faecal homogenate at $105^{\circ} \mathrm{C}$ for $12 \mathrm{~h}$. Crucibles were allowed to cool and were then weighed, and moisture content was calculated by difference.

Ammonia content in the faecal homogenates was determined according to a colorimetric method ${ }^{(24)}$. The results were expressed as $\mu \mathrm{mol} / \mathrm{g}$ of faeces. TAC of properly diluted faecal water was estimated from its ability to reduce a $\mathrm{Fe}$ (III)-2,4,6-tri(2-pyridyl)-s-triazine complex to $\mathrm{Fe}(\mathrm{II})-2,4$, 6-tri(2-pyridyl)-s-triazine by using a multiplate reader (Tecan, Maennedorf, Switzerland) (ferric reducing antioxidant power assay) ${ }^{(23)}$. Each determination was performed in triplicate.

Total polyphenol content of faecal water was determined by the Folin-Ciocalteu assay ${ }^{(25)}$.

Single-cell gel electrophoresis assay (COMET assay) was carried out in HT-29 cells for the assessment of faecal water genotoxicity $^{(26)}$.

\section{Quantification of Lactobacillus and Bifidobacterium by real-time $P C R$}

Lactobacillus and Bifidobacterium genomic DNA in the faecal homogenates were quantified by real-time PCR (RT-PCR). RT-PCR was performed on the LightCycler System (Roche Applied Science, Mannheim, Baden-Württemberg, Germany) using LightCycler FastStart DNA Master ${ }^{\text {PLUS }}$ SYBR Green I (Roche Applied Science). The fragment of the 16S rRNA gene specific for Lactobacillus and Bifidobacterium genera was amplified using primers ${ }^{(27,28)}$. The $20 \mu$ l total reaction volume was composed of the following: $4 \mu \mathrm{l}$ of LightCycler FastStart DNA Master PLUS SYBR Green I (Roche Diagnostics), $0.5 \mu \mathrm{M}$ forward and reverse primers and $5 \mu \mathrm{l}$ of extracted DNA. The amplification programme included an initial denaturation step at $95^{\circ} \mathrm{C}$ for $10 \mathrm{~min}$ followed by forty cycles of denaturation for $10 \mathrm{~s}$ at $95^{\circ} \mathrm{C}$, annealing for $20 \mathrm{~s}$ at $60^{\circ} \mathrm{C}$ and extension for $5 \mathrm{~s}$ at $72^{\circ} \mathrm{C}$. The temperature transition rate was $20^{\circ} \mathrm{C} / \mathrm{s}$.

For the melting curve analysis, the samples were denatured at $95^{\circ} \mathrm{C}$ for $0 \mathrm{~s}$, cooled at $65^{\circ} \mathrm{C}$ for $15 \mathrm{~s}$ and then slowly heated to $95^{\circ} \mathrm{C}$ at a temperature transition rate of $0.1{ }^{\circ} \mathrm{C} / \mathrm{s}$ with continuous monitoring of fluorescence. Genomic DNA from Lactobacillus brevis (ATCC 8287) and Bifidobacterium infantis (ATCC 15697) were purchased from the American Type Culture Collection (ATCC, Manassas, VA, USA), and were used for optimising RT-PCR and generating quantification standards. For the construction of standard curves, tenfold dilution series of between $0.01 \mathrm{pg}$ and $10 \mathrm{ng}$ from target species genomic DNA were used. The standard curves of RT-PCR were used for the quantification of the target bacterial DNA from faecal DNA preparations, and PCR results were converted to the average estimate of target bacterial genomes present in $1 \mathrm{~g}$ of faeces (wet weight).
RT-PCR was applied for detecting two bacterial genera that include several species with different ribosomal gene copy numbers and genome size. For this reason, we used the estimated average genome sizes and we did not consider the differences in the copy number of $16 \mathrm{~S}$ rRNA gene, as suggested by Malinen et al. ${ }^{(28)}$. The estimated average genome sizes for Lactobacillus and Bifidobacterium used for converting results obtained by RT-PCR were 2.3 and $2 \mathrm{Mb}$, respectively. Three parallel PCR for each sample were analysed.

Standard curves had correlation coefficient values of between 0.99 and 1 , while the amplification slope was between -3.35 and -3.39 . The standard curves of RT-PCR were used for the quantification of the target bacterial DNA from faecal DNA preparations, and the PCR results were converted to the average estimate of target bacterial genomes present in $1 \mathrm{~g}$ of faeces (wet weight). The estimated average genome sizes for Lactobacillus and Bifidobacterium used for converting results obtained by RT-PCR were 2.3 and $2 \mathrm{Mb}$, respectively. Three parallel PCR for each sample were analysed.

\section{Statistical analysis}

Dietary, clinical and biological variables were tested for normality with the Kolmogorov-Smirnov test. Dietary variables were normally distributed and differences among HT, LT and WO periods were assessed using the repeated-measures General Linear Model and the Bonferroni test for post hoc comparisons, with diet being considered as a within-subject factor. Between-diet (LT and HT) comparisons were performed using paired Student's $t$ tests for variables normally distributed (moisture and ammonia), and the Wilcoxon test for paired samples for variables non-normally distributed (faecal $\mathrm{pH}$, faecal weight and content of TAC and total polyphenols). Significance level was set at $P<0 \cdot 05$. Statistical analyses were performed using Statistical Package for Social Sciences (version 16.0; SPSS, Inc., Chicago, IL, USA).

\section{Results}

\section{Subjects and diet}

One subject withdrew immediately after randomisation for reasons unrelated to the study. Therefore, nineteen subjects (ten females) completed the study (age 60 (SD 3) years, BMI $26.2(\mathrm{SD} 2.5) \mathrm{kg} / \mathrm{m}^{2}$ ). A subset of nine subjects (five females) successfully completed the additional part of the protocol for the evaluation of markers of bowel function (age 59 (SD 3) years, BMI $26.4(\mathrm{SD} 2 \cdot 9) \mathrm{kg} / \mathrm{m}^{2}$ ) (Table 1).

Dietary intakes during HT, LT and WO periods are summarised in Table 2. Dietary data obtained during the WO period are considered representative of the subjects' habitual diet. As per protocol, total energy, macronutrient and alcohol intake did not differ significantly during each study period. Total fibre intake did not differ between HT and LT periods, and it was significantly higher in either one than in the WO period $(P=0 \cdot 001)$.

The selection of HT foods led to significantly higher intakes of TAC, vitamin $\mathrm{C}$, catechins and flavanones during the HT period than during both the LT and WO periods $(P<0 \cdot 001)$. 
Table 1. Baseline characteristics for the whole group of subjects and the subset of nine subjects with complete repeated faecal collection (Mean values and standard deviations)

\begin{tabular}{|c|c|c|c|c|}
\hline & \multicolumn{2}{|c|}{ All $(n 19)$} & \multicolumn{2}{|c|}{$\begin{array}{l}\text { Subset with full } \\
\text { data set }(n 9)\end{array}$} \\
\hline & Mean & $\overline{S D}$ & Mean & SD \\
\hline Males $(n)$ & \multicolumn{2}{|c|}{9} & \multicolumn{2}{|c|}{4} \\
\hline Females $(n)$ & \multicolumn{2}{|c|}{10} & \multicolumn{2}{|c|}{5} \\
\hline Age (years) & 60 & 3 & 59 & 3 \\
\hline Height $(m)$ & 1.62 & 0.08 & 1.62 & $0.0 s$ \\
\hline Weight (kg) & $69 \cdot 0$ & 9.8 & $69 \cdot 3$ & 9.3 \\
\hline $\operatorname{BMI}\left(\mathrm{kg} / \mathrm{m}^{2}\right)$ & $26 \cdot 2$ & 2.5 & $26 \cdot 4$ & 2.9 \\
\hline Waist girth $(\mathrm{cm})$ & 93.3 & $7 \cdot 7$ & 93.6 & $7 \cdot 6$ \\
\hline
\end{tabular}

$M$, male; $F$, female.

During the HT diet, the intake of vitamin E, anthocyanins and lignans was significantly higher than that in the LT period $(P<0 \cdot 001)$, but it was not different from that in the WO period, whereas the intake of $\beta$-carotene did not differ significantly between the HT and LT periods, but it was significantly higher than that in the WO period. In the HT period, flavones, flavonols, isoflavones and pro-anthocyanidins were either not different or lower than those in the LT and WO periods.

During the HT period, the increase in daily TAC intake with respect to the WO period was primarily due to higher TAC intake from fruits and vegetables, whereas TAC intake from alcoholic beverages, tea and coffee remained almost unchanged. Conversely, during the LT period, the reduction in daily TAC intake was mainly due to a lower TAC intake from such beverages compared with the WO period. The main contributors to daily TAC intake in the three dietary periods were alcoholic beverages $(20 \%$ in $\mathrm{HT}, 26 \%$ in WO and $18 \%$ in LT, with red wine being the major contributor), coffee (26\% in HT, $38 \%$ in WO and $3 \%$ in LT), and fruits and vegetables (34\% in HT, $23 \%$ in WO and $41 \%$ in LT).

Daily TAC intake from alcoholic beverages and coffee was more than ten times higher for the HT diet than for the LT diet $(+1104 \%)$. Fruit and vegetable contribution to TAC in HT diet was $350 \%$ higher than that in the LT diet (data not shown). These indications suggest that the dietary advice followed while selecting food items with different TAC in HT and LT diets effectively met the study objectives.

\section{Effect of diet on bowel function}

Data on bowel function are summarised in Tables 2 and 3 for all the subjects and for subjects who completed the repeated faecal collection, respectively.

Switching to antioxidant-rich foods did not affect the frequency of evacuation or consistency of stools, neither as self-graded by the subjects (data not shown) nor when measured as moisture content in $48 \mathrm{~h}$ faecal samples in the subset of subjects (Table 3). Faecal moisture was in the normal range ${ }^{(5)}$. Faecal $\mathrm{pH}$ before and after either diet

Table 2. Dietary intake and faecal output for all the subjects ( $n$ 19)

(Mean values with their standard errors)

\begin{tabular}{|c|c|c|c|c|c|c|c|}
\hline & \multicolumn{2}{|c|}{ LT } & \multicolumn{2}{|c|}{$\mathrm{HT}$} & \multicolumn{2}{|c|}{ wo } & \multirow[b]{2}{*}{$P^{*}$} \\
\hline & Mean & SEM & Mean & SEM & Mean & SEM & \\
\hline \multicolumn{8}{|l|}{ Energy, nutrient and food intake } \\
\hline Total energy (kJ) & 9894 & 397 & 10279 & 410 & 10132 & 359 & 0.436 \\
\hline Energy from fat (\%) & $34 \cdot 1$ & $1 \cdot 2$ & 31.9 & 1.3 & 34.8 & 1.0 & $0 \cdot 108$ \\
\hline Energy from carbohydrate (\%) & $47 \cdot 3$ & 1.5 & $49 \cdot 8$ & $1 \cdot 3$ & $46 \cdot 3$ & $1 \cdot 2$ & 0.106 \\
\hline Energy from protein (\%) & $14 \cdot 7$ & 0.4 & $14 \cdot 7$ & 0.6 & $14 \cdot 3$ & 0.5 & 0.598 \\
\hline Alcohol (g) & $22 \cdot 8$ & $2 \cdot 4$ & $22 \cdot 7$ & 3.6 & $25 \cdot 2$ & 4.0 & 0.607 \\
\hline Total dietary fibre (g) & $25 \cdot 4^{\mathrm{a}}$ & 1.4 & $24 \cdot 9^{a}$ & 1.2 & $22 \cdot 0^{\mathrm{b}}$ & $1 \cdot 1$ & 0.001 \\
\hline Bread, pasta and cereals ( $\mathrm{g}$ ) & 233 & 16 & 216 & 18 & 231 & 22 & 0.774 \\
\hline Fruits and vegetables $(\mathrm{g})$ & $648^{a}$ & 32 & $665^{\mathrm{a}}$ & 46 & $494^{\mathrm{b}}$ & 33 & $<0.001$ \\
\hline Coffee and tea $(\mathrm{g})$ & $25^{\mathrm{a}}$ & 8 & $158^{\mathrm{b}}$ & 21 & $118^{\mathrm{b}}$ & 16 & $<0.001$ \\
\hline \multicolumn{8}{|l|}{ Antioxidant intake } \\
\hline TAC (mmol FRAP) & $5 \cdot 4^{\mathrm{a}}$ & 1.5 & $29 \cdot 3^{b}$ & $9 \cdot 1$ & $17 \cdot 8^{\mathrm{c}}$ & $5 \cdot 5$ & $<0.001$ \\
\hline Vitamin C $(\mathrm{mg})$ & $74^{a}$ & 5 & $438^{\mathrm{b}}$ & 51 & $139^{c}$ & 17 & $<0.001$ \\
\hline Vitamin E (mg) & $7 \cdot 6^{\mathrm{a}}$ & 0.4 & $16 \cdot 3^{\mathrm{b}}$ & 1.2 & $15 \cdot 3^{\mathrm{b}}$ & 1.0 & $<0.001$ \\
\hline$\beta$-Carotene (mg) & $3 \cdot 9^{\mathrm{a}, \mathrm{b}}$ & 0.5 & $4 \cdot 2^{\mathrm{a}}$ & 0.4 & $2 \cdot 8^{\mathrm{b}}$ & 0.4 & 0.036 \\
\hline Catechins (mg) & $30^{\mathrm{a}}$ & 13 & $63^{b}$ & 22 & $35^{\mathrm{a}}$ & 13 & $<0.001$ \\
\hline Flavones (mg) & 0.8 & 1.3 & 1.2 & $2 \cdot 0$ & $1 \cdot 1$ & 1.3 & 0.660 \\
\hline Flavanones (mg) & $1 \cdot 1^{\mathrm{a}}$ & 3.0 & $124^{b}$ & 51 & $34^{\mathrm{c}}$ & 42 & $<0.001$ \\
\hline Flavonols (mg) & $48^{\mathrm{a}}$ & 40 & $14^{\mathrm{b}}$ & 10 & $23^{a, b}$ & 36 & 0.005 \\
\hline Isoflavones (mg) & $475^{\mathrm{a}}$ & 284 & $418^{\mathrm{a}, \mathrm{b}}$ & 223 & $330^{\mathrm{b}}$ & 131 & 0.005 \\
\hline Anthocyanins (mg) & $0.2^{\mathrm{a}}$ & 0.1 & $30^{\mathrm{b}}$ & 21 & $16^{\mathrm{b}}$ & 14 & $<0.001$ \\
\hline Lignans (mg) & $70^{\mathrm{a}}$ & 33 & $518^{b}$ & 430 & $300^{\mathrm{b}}$ & 206 & $<0.001$ \\
\hline Pro-anthocyanidins (mg) & 312 & 107 & 260 & 137 & 231 & 140 & 0.123 \\
\hline \multicolumn{8}{|l|}{ Faecal output } \\
\hline Average $48 \mathrm{~h}$ faeces wet weight $(\mathrm{g})$ & $218^{\mathrm{a}}$ & 22 & $324^{\mathrm{b}}$ & 38 & $216^{\mathrm{a}}$ & 24 & 0.038 \\
\hline Average daily bowel movements $(n)$ & 1.05 & 0.05 & 1.04 & 0.06 & 1.00 & 0.04 & 0.678 \\
\hline
\end{tabular}

LT, low-total antioxidant capacity; HT, high-total antioxidant capacity; WO, wash-out; TAC, total antioxidant capacity; FRAP, ferric reducing antioxidant power.

a,b,c Mean values within a row with unlike superscript letters were significantly different $(P<0.05)$.

${ }^{*} P$ value for differences between means (repeated measures: general linear model with the Bonferroni correction for multiple comparisons). 
intake remained unchanged, and was not significantly different between the HT and LT diets. No significant change was observed in endogenous ammonia before and after either diet intake, or between interventions.

Despite the fact that stool consistency did not change, mean weight of the faeces evacuated over $48 \mathrm{~h}$ was significantly higher at the end of the HT period than at the end of both the LT and WO periods $(P=0.038)$.

\section{Effect of diet on faecal water antioxidants and toxicity}

Increased intake of dietary antioxidants led to higher TAC of faecal waters, expressed both as the concentration of ferric reducing antioxidant power $(\mathrm{mmol} / \mathrm{l}$ faecal water; $P=0.011$ ) and as ferric reducing antioxidant power excreted over $48 \mathrm{~h}$ (mmol; $P=0.011$ ), at the end of the HT period than at the end of the LT period (Table 4).

Despite the fact that the increased intake of total phenols in the HT diet did not reach statistical significance compared with the LT diet (data not shown), total phenols in faeces assessed by the Folin-Ciocalteu method were higher in the HT diet than in the LT diet $(P=0.008$ for total phenols expressed as $\mathrm{mmol} / \mathrm{l} ; P=0.011$ for total phenols expressed as $\mathrm{mmol} / 48 \mathrm{~h}$ ) (Table 4$)$.

Faecal water genotoxicity, measured as tail intensity using the alkaline COMET assay, was analysed after HT and LT periods, which showed high inter-individual variability and no significant differences between the dietary periods (data not shown).

\section{Faecal microbiota}

Fig. 1 shows the variation in Lactobacillus and Bifidobacterium counts in stool samples and excreted cells over $48 \mathrm{~h}$ after both the HT and LT diet intakes. Despite a trend in the reduction in the number of bacterial cells after the HT diet intake, no significant changes were observed in Lactobacillus or Bifidobacterium counts during either dietary intervention, or between diets.

\section{Discussion}

In intervention studies aimed at investigating the impact of dietary antioxidants on health markers, the TAC can be regarded as a comprehensive parameter that is able to describe the quality of diets with respect to antioxidant content and activity. In the present study, subjects based their food choices on a list of either HT or LT fruits, vegetables, beverages and condiments ${ }^{(14,15)}$. The approach successfully led to a significant increase in overall dietary TAC in the HT period than in the LT period, while energy, macronutrients, alcohol and total dietary fibre did not differ. The fact that the intake of total dietary fibre did not differ between the intervention periods is probably the result of consumption of the same number of portions of fruits, vegetables and cereals in both diets. This is of paramount importance as total fibre intake is the dietary factor that is most able to affect the bowel function ${ }^{(29)}$.

The present results indicate that despite the introduction of similar quantities of macronutrients and total dietary fibre, the faecal output is significantly higher after the consumption of a diet rich in antioxidants. This is the first time that a human 
Table 4. Faecal antioxidants in the subset of nine subjects with complete repeated faecal collection (Median values and interquartile ranges)

\begin{tabular}{|c|c|c|c|c|c|}
\hline & \multicolumn{2}{|c|}{ Post LT } & \multicolumn{2}{|c|}{ Post HT } & \multirow[b]{2}{*}{$P^{*}$} \\
\hline & Median & $\begin{array}{l}\text { Interquartile } \\
\text { range }\end{array}$ & Median & $\begin{array}{l}\text { Interquartile } \\
\text { range }\end{array}$ & \\
\hline Average stools excreted ( $g / 48 \mathrm{~h}$ ) & 175 & 154 & 358 & 338 & 0.015 \\
\hline TAC in faecal water $(\mathrm{mmol} / \mathrm{l})$ & $39 \cdot 37$ & 34.36 & 49.02 & $29 \cdot 27$ & 0.011 \\
\hline Total TAC excreted (mmol/48 h) & $6 \cdot 13$ & $4 \cdot 14$ & $14 \cdot 77$ & $12 \cdot 83$ & 0.011 \\
\hline Phenol concentration in faecal water $(\mathrm{mmol} / \mathrm{l})$ & $7 \cdot 67$ & $6 \cdot 46$ & $13 \cdot 70$ & 8.72 & 0.008 \\
\hline Total phenols excreted $(\mathrm{mmol} / 48 \mathrm{~h})$ & 1.83 & 1.13 & 4.26 & 3.63 & 0.011 \\
\hline
\end{tabular}

LT, low-total antioxidant capacity; HT, high-total antioxidant capacity; TAC, total antioxidant capacity.

${ }^{\star} P$ value for differences between treatments (Wilcoxon test for paired samples).

intervention has shown the efficacy of dietary antioxidants in achieving greater stool weight. Other determinants of bowel habit, such as frequency of evacuation and stool consistency, did not change. Previous observations show that in the pool of dietary antioxidants, the bioavailability varies broadly, with carotenoids and tocopherols being more available in the small intestine for absorption ${ }^{(9)}$, and polyphenols most likely to transit undigested. In the present study, the higher TAC in the HT diet was indeed supported by a higher intake of phenolics such as anthocyanins, other flavonoids and lignans. The main contributors of TAC were fibre-poor beverages, such as red wine and coffee, which are notably polyphenol rich ${ }^{(10)}$. Faecal bulking effects, such as the one observed in the present study, are usually ascribed to dietary fibre, and are considered to be linked to a spectrum of health effects in the large intestine ${ }^{(30)}$. Stool weight below $150 \mathrm{~g} / \mathrm{d}$ is associated with greater risk for colon cancer ${ }^{(31)}$. While daily

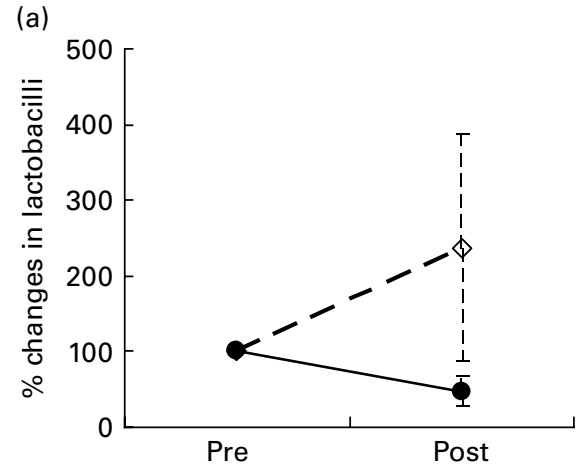

(c)

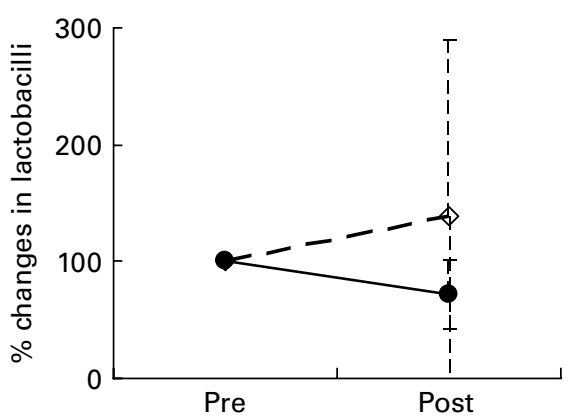

stool weight at the end of the LT period was below this threshold, after the HT period, it doubled and exceeded the critical $150 \mathrm{~g} / \mathrm{d}$.

Data on faecal specimens obtained from nine subjects confirm that a certain proportion of antioxidants from the diet did reach the colon, as documented by the higher TAC and total polyphenols content of their faecal waters at the end of the HT period. However, no difference was observed in the faecal moisture or in the volume of faecal water between HT and LT diets, indicating that the poorly absorbed polyphenols deriving from the HT diet did not enhance the water-holding capacity, in contrast with what has been observed previously in rats ${ }^{(12)}$. Moreover, no effect was observed in faecal water genotoxicity (data not shown), indicating that faecal polyphenols, in the conditions given, were not able to protect epithelial cells from oxidative or mutagenic insults in vitro. (b)

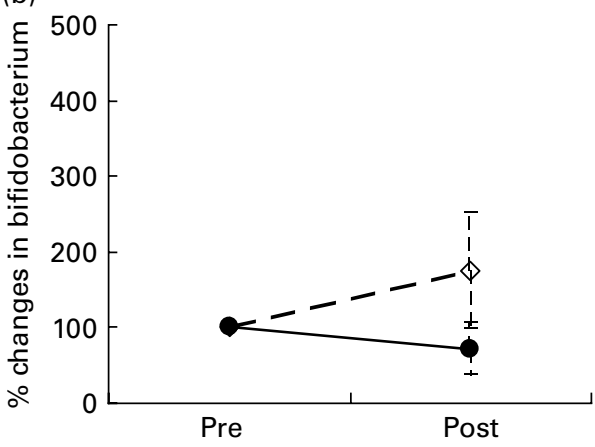

(d)

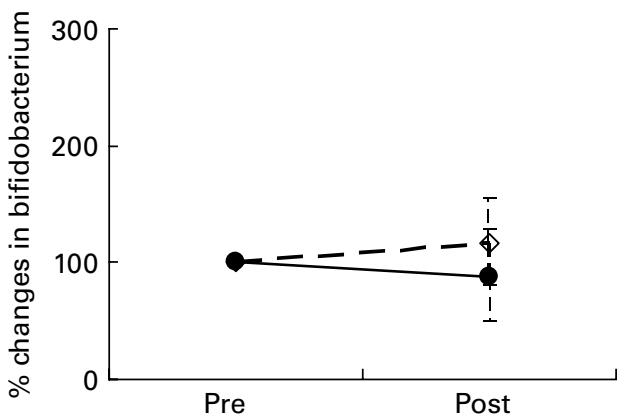

Fig. 1. Percentage changes in lactobacilli ((a) and (c)) and bifidobacteria ((b) and (d)) after the consumption of low-total antioxidant capacity (- $\diamond-)$ or high-total antioxidant capacity (- - ) diets. (a) and (b) refer to bacterial counts (no. of bacterial cells/g faeces); (c) and (d) refer to total $48 \mathrm{~h}$ excretion of bacteria ( $n$ of bacterial counts corrected for faecal mass). 
A possible explanation for the faecal bulking effect of polyphenol-rich foods might reside in their capacity to inhibit bacterial activity and thus fibre fermentation, which per se can reduce faecal output. In general, polyphenols are a wide group of substances, and biological effects may differ between compounds on the basis of molecular weight, water solubility and ability to chelate minerals or to form complexes with proteins, thus inhibiting the enzyme activity to various degrees. This is well known in animal nutrition, and can be regarded as either negative or positive depending on the type and amount of phenolics fed with forages ${ }^{(32)}$. The effect of undigested polyphenols and their fermentation metabolites on the human faecal microbiota is more controversial, with studies reporting a growth repression of both $\operatorname{Gram}(+)$ and $\operatorname{Gram}(-)$ bacteria $^{(33)}$, and others indicating a milder inhibition or an increase in Bifidobacterium spp. ${ }^{(34,35)}$. In a recent study, Jaquet et al. ${ }^{(36)}$ observed that the consumption of coffee produced an increase in the metabolic activity and/or numbers of the Bifidobacterium spp. population without a major impact on the dominant microbiota. However, they could not discriminate which coffee component was responsible for this effect (polyphenols or fibres present in coffee). In the present study, when the population of intestinal lactobacilli and bifidobacteria were quantified, no significant changes were found in the microbial community of individuals before and after HT or LT period, but indeed a tendency to lower bacterial concentrations after HT period was noticed, possibly indicating an antibiotic (as opposed to prebiotic) effect of polyphenols on these bacterial genera. However, the quantification of SCFA would have been a more direct approach to assess the modulation of fermentation activities in the gut by the dietary intervention. Similarly, the quantification of protein, fat and fibre in the faecal samples would have been of help to clarify the hypothetical mechanisms by which phenols induce a higher stool output. Another limitation of the present study is that the characterisation of faecal microbiota was a priori restricted to probiotic genera, such as Lactobacillus and Bifidobacterium, based on their putative beneficial effect on gut health ${ }^{(37)}$, and no commensal or pathogenic microorganisms were quantified. Additionally, a longer intervention time, exceeding the $14 \mathrm{~d}$ of the present study, would have been advisable to investigate whether significant changes in the composition of microbiota occurred. Finally, and in order to exclude an effect of differences in the composition of dietary fibre consumed during the two intervention periods on stool weight, the relative contribution of different types of fibres with different bulking effects (i.e. soluble and insoluble) to total fibre intake should have been evaluated. However, since the same amount of fruits and vegetables was consumed during each intervention (LT and HT) period, and subjects followed their usual diet regarding the consumption of cereal products throughout the study, significant differences between intervention periods regarding the type of fibre preferentially consumed are unlikely.

In conclusion, the present study indicates that HT diets lead to an increase in antioxidant content of faeces. This is accompanied by a significant increase in stool bulk, a factor considered to be of potential relevance for bowel health. The mechanism of action remains speculative, but it might be mediated by an effect of dietary polyphenols on colonic microbiota. High polyphenol intakes can be achieved through the selection of HT food items without significantly changing the dietary habits.

\section{Acknowledgements}

The study was supported by COFIN 2004 project from the Italian Ministry of University and Research and by the EC project 'PIPS - Personalised Information Platform for Life and Health Services' (IST-2004-507019). The contributions of the authors are as follows: F. S., S. V., L. F., D. D. R. and M. A. B. were involved in the requisition of approval from the ethics committee, subject recruitment, collection and interpretation of the clinical data, and subjects' management; I. Z. was responsible for the requisition of approval from the ethics committee, subject recruitment, collection and interpretation of the clinical data and subjects' management; M. A. B., F. S., D. D. R. and N. P. were responsible for the collection and interpretation of dietary and laboratory data; C. P., A. B. and M. L. C. were responsible for the analysis and interpretation of biological data; M. A. B., F. S., D. D. R. and F. B. were the primary authors of the manuscript, and were responsible for study concept and design, and all other authors provided input; F. B. and I. Z. secured the funding for the study. None of the authors has conflicts of interest (financial, professional or personal) that are relevant to the study.

\section{References}

1. Joint WHO/FAO Expert Consultation on Diet, Nutrition and the Prevention of Chronic Diseases (2003) Diet, Nutrition and the Prevention of Chronic Diseases: Report of a Joint WHO/FAO Expert Consultation. WHO Technical Report Series no. 916. Geneva: WHO.

2. Hung H-C, Joshipura KJ, Jiang R, et al. (2004) Fruit and vegetable intake and risk of major chronic disease. $J$ Natl Cancer Inst 96, 1577-1584.

3. Willcox JK, Ash S \& Catignani GL (2004) Antioxidants and prevention of chronic disease. Crit Rev Food Sci Nutr 44, 275-295.

4. Burkitt DP, Walker ARP \& Painter NS (1972) Effect of dietary fibre on stools and transit-times, and its role in the causation of disease. Lancet ii, 1408-1412.

5. Cumming JH, Antoine J-M, Azpiroz F, et al. (2004) PASSCLAIM - gut health and immunity. Eur J Nutr 43, 118-173.

6. Garsetti M, Pellegrini N, Baggio C, et al. (2000) Antioxidant activity in human faeces. Br J Nutr 84, 705-710.

7. Bingham SA, Vorster H, Jerling JC, et al. (1997) Effect of black tea drinking on blood lipids, blood pressure and aspects of bowel habit. Br J Nutr 73, 531-550.

8. Schnäbele K, Briviba K, Bub A, et al. (2008) Effects of carrot and tomato juice consumption on fecal markers relevant to colon carcinogenesis in humans. Br J Nutr 99, 606-613.

9. Goi I, Serrano J \& Saura-Calixto F (2006) Bioaccessibility of $\beta$-carotene, lutein, and lycopene from fruits and vegetables. J Agric Food Chem 54, 5382-5387.

10. Clifford MN (2004) Diet-derived phenols in plasma and tissues and their implications for health. Planta Med 70, 1103-1114.

11. Jenner AM, Rafter J \& Halliwell B (2005) Human fecal water content of phenolics: the extent of colonic exposure to aromatic compounds. Free Radic Biol Med 38, 763-772. 
12. Bravo E, Mañas E \& Saura-Calixto F (1993) Dietary nonextractable condensed tannins as indigestible compounds: effects on fecal weight, and protein and fat excretion. $J$ Sci Food Agric 63, 63-68.

13. Bravo L, Abia R, Eastwood MA, et al. (1994) Degradation of polyphenols (catechin and tannic acid) in the rat intestinal tract. Effect on colonic fermentation and fecal output. $\mathrm{Br} J$ Nutr 71, 933-946.

14. Pellegrini N, Serafini M, Colombi B, et al. (2003) Total antioxidant capacity of plant foods, beverages and oils consumed in Italy assessed by three different in vitro assays. $J$ Nutr 133, 2812-2819.

15. Pellegrini N, Serafini M, Salvatore S, et al. (2006) Total antioxidant capacity of spices, dried fruits, nuts, pulses, cereals and sweets consumed in Italy assessed by three different in vitro assays. Mol Nutr Food Res 50, 1030-1038.

16. Dudonné S, Vitrac X, Coutière P, et al. (2009) Comparative study of antioxidant properties and total phenolic content of 30 plant extracts of industrial interest using DPPH, ABTS, FRAP, SOD and ORAC assays. J Agric Food Chem 57, $1768-1774$.

17. Wu X, Pittman HE \& Prior RL (2006) Fate of anthocyanins and antioxidant capacity in contents of the gastrointestinal tract of weanling pigs following black raspberry consumption. J Agric Food Chem 54, 583-589.

18. Gibson G \& Robertfroid MB (1995) Dietary modulation of the human colonic microbiota: introducing the concept of prebiotics. J Nutr 125, 1401-1412.

19. Halliwell B, Rafter J \& Jenner A (2005) Health promotion by flavonoids, tocopherols, tocotrienols, and other phenols: direct or indirect effects? Antioxidant or not? Am J Clin Nutr 81, Suppl. 1, 268S-276S.

20. Pearson J, Gill C \& Rowland I (2009) Diet, fecal water, and colon cancer-development of a biomarker. Nutr Rev 67, 509-526.

21. Valtueña S, Pellegrini N, Franzini L, et al. (2008) Food selection based on total antioxidant capacity can modifiy antioxidant intake, systemic inflammation, and liver function without altering markers of oxidative stress. Am J Clin Nutr 87, 1290-1297.

22. Salvini S (1987) A food composition database for epidemiological studies in Italy. Cancer Lett 114, 299-300.

23. Benzie IF \& Strain JJ (1996) The ferric reducing ability of plasma (FRAP) as a measure of "antioxidant power": the FRAP assay. Anal Biochem 239, 70-76.
24. Hughes R, Kurth MJ, McGilligan V, et al. (2008) Effect of colonic bacterial metabolites on Caco-2 cell paracellular permeability in vitro. Nutr Cancer 60, 259-266.

25. Singleton VL \& Rossi JA (1965) Colorimetry of total phenolics with phosphomolybdic-phosphotungstic acid reagents. Am J Enol Vitic 16, 144-158.

26. Venturi M, Hamby RJ, Glinghammar B, et al. (1997) Genotoxic activity in human fecal water and the role of bile acids: a study using the alkaline comet assay. Carcinogenesis 12, 2353-2359.

27. Penders J, Vink C, Driessen C, et al. (2005) Quantification of Bifidobacterium spp., Echerichia coli and Clostridium difficile in fecal samples of breast-fed and formula-fed infants by realtime PCR. FEMS Microbiol Lett 243, 141-147.

28. Malinen E, Rinttilä T, Kajander K, et al. (2006) Analysis of the fecal microbiota of irritable bowel syndrome patients and healthy controls with real-time PCR. Am J Gastroenterol 100, 373-382.

29. Cummings JH \& Macfarlane GT (2002) Gastrointestinal effects of prebiotics. Br J Nutr 87, 145-151.

30. Schneeman BO (2001) Dietary fibre and gastrointestinal function. In Advanced Dietary Fibre Technology, pp. 168-176 [BV McLeary and L Prosk, editors]. Oxford: Blackwell Science Limited.

31. Cummings JH, Bingham SA, Heaton KW, et al. (1992) Fecal weight, colon cancer risk, and dietary intake of nonstarch polysaccharides (dietary fibre). Gastroenterology 103, $1783-1789$.

32. Reed JD (1995) Nutritional toxicology of tannins and related polyphenols in forage legumes. J Anim Sci 73, 1516-1528.

33. Friedman M (2007) Overview of antibacterial, antitoxin, antiviral, and antifungal activities of tea flavonoids and teas. Mol Nutr Food Res 51, 116-134.

34. Okubo $\mathrm{T}$, Ishihara $\mathrm{N}$, Oura A, et al. (1992) In vivo effects of tea polyphenol intake on human intestinal microflora and metabolism. Biosci Biotechnol Biochem 56, 588-591.

35. Cheng Lee H, Jenner AM, Seng Low C, et al. (2006) Effect of tea phenolics and their aromatic fecal bacterial metabolites on intestinal microbiota. Res Microbiol 157, 876-884.

36. Jaquet M, Rochat I, Moulin J, et al. (2009) Impact of coffee consumption on the gut microbiota: a human volunteer study. Int J Food Microbiol 130, 117-121.

37. Floch M, Walker A, Guandalini S, et al. (2008) Recommendations for probiotic use. J Clin Gastroenterol 42, Suppl. 2, S104-S108. 